\title{
Identification et distribution géographique des virus responsables des mosaïques chez les Cucurbitacées en Côte d'Ivoire
}

\author{
Kouakou Théodore KOUADIO*, Thérèse ATCHAM AGNEROH, \\ Kolotcholohofolo SORO et Aristide GONE \\ Institut National Polytechnique Félix Houphouët-Boigny, Département de Formation et de Recherche \\ Agriculture et Ressources Animales, Laboratoire de Phytopathologie et de Biologie Végétale, \\ B.P. 1313 Yamoussoukro- Côte d'Ivoire. \\ *Auteur correspondant ; E-mail: tkouadiothed@gmail.com; Tél : (+225) 49999445
}

\section{RESUME}

Les viroses constituent une menace pour la réussite des Cucurbitacées de par leurs effets néfastes sur les rendements et la valeur marchande de la production. L'objectif de ce travail était d'identifier les virus responsables de symptômes de mosaïques dans les principales zones de production de Cucurbitacées en Côte d'Ivoire. Une enquête visant à détecter quatre virus majeurs (Cucumber Mosaic Virus (CMV), Papaya Ringspot Virus (PRSV), Watermelon Mosaic Virus (WMV) et Zucchini Yellow Mosaic Virus (ZYMV)) a été menée dans 18 parcelles de Cucumis sativus, Cucurbita maxima et Cucurbita pepo localisées à Abidjan, Bouaké, Daloa, Korhogo, Man, San Pedro et Yamoussoukro. Les tests sérologiques DAS-ELISA réalisés sur les 169 échantillons collectés ont montré la présence du ZYMV, CMV, WMV et PRSV avec respectivement $59,76 \% ; 34,91 \% ; 10,65 \%$ et $10,65 \%$. Au total, 136 échantillons sur les 169 se sont révélés porteurs d'au moins un des quatre virus testés. Les infections mixtes les plus importantes sont celles entre le CMV et le ZYMV avec un taux d'infection de $14,79 \%$ sur l'ensemble des échantillons testés. Par ailleurs, cette étude rapporte pour la première fois le WMV en Côte d'Ivoire. Ces résultats constituent une première étape en vue de parvenir à une gestion intégrée durable des virus des Cucurbitacées en Côte d'Ivoire.

(C) 2017 International Formulae Group. All rights reserved.

Mots clés : CMV, PSRV, WMV, ZYMV, DAS-ELISA, Côte d'Ivoire.

\section{Identification and geographic distribution of viruses causing mosaic diseases on Cucurbitaceae in Côte d'Ivoire}

\begin{abstract}
Virus diseases are considered as a potential threat to Cucurbitaceae productions because of their serious adverse effects on crop yields and market value. The study aimed at identifying viruses causing mosaic diseases in the major production regions in Ivory Coast. A survey aimed at detecting four major viruses of Cucurbitaceae in Ivory Coast (Cucumber mosaic virus (CMV), Papaya ringspot virus (PRSV), Watermelon mosaic virus (WMV) and Zucchini yellow mosaic virus (ZYMV)) was carried out in 18 plots of Cucumis sativus, Cucurbita maxima and Cucurbita pepo across seven regions of the country (Abidjan, Bouaké, Daloa, Korhogo, Man, San Pedro and Yamoussoukro). Of the 169 samples collected, we could detect from serological tests DAS-ELISA the presence of ZYMV, CMV, WMV and PRSV with respectively infection rate of 59.76\%;
\end{abstract}


$34.91 \% ; 10.65 \%$ and $10.65 \%$. A total of 136 samples out of 169 tested positive to at least one of the four viruses studied. The largest mixed infections are those CMV+ZYMV with an infection rate of $14.8 \%$ for all the samples tested. This study reports for the first time the presence of WMV on Cucurbitaceae in Ivory Coast. The results of this work constitute a first step in taking into account a sustainable integrated management of viruses on Cucurbitaceae in Côte d'Ivoire.

(C) 2017 International Formulae Group. All rights reserved.

Keywords: CMV, PSRV, WMV, ZYMV, DAS-ELISA, Ivory Coast.

\section{INTRODUCTION}

Dans l'agriculture vivrière de la Côte d'Ivoire, les cultures maraîchères et notamment les Cucurbitacées représentent une importante source de lipides, de protéines ou de glucides dans l'alimentation. Elles constituent aussi une source de revenus pour certains groupes vulnérables notamment les femmes et les jeunes déscolarisés engagés dans le maraîchage (ANADER, 2004 ; FAO, 2009). Malgré cette importance économique, les Cucurbitacées sont sujettes à plusieurs ravageurs et maladies dont les plus dommageables sont celles d'origine virale (Provvidenti, 1996; Ali et al., 2012a). Plus d'une trentaine de virus peuvent infecter naturellement les Cucurbitacées à travers le monde (Mnari et al., 2008; Provvidenti, 2010; Ali et al., 2012a). Ils causent des symptômes de mosaïques et de boursouflures sur les feuilles, des fruits difformes et des plants rabougris (Ali et al., 2004 ; Verma et al., 2006 ; Mnari et al., 2008). Les virus responsables de mosaïque en l'occurrence le Cucumber mosaic virus (CMV, Cucumovirus), le Papaya ringspot virus (PRSV, Potyvirus), le Watermelon mosaic virus (WMV, Potyvirus) et le Zucchini yellow mosaic virus (ZYMV, Potyvirus) ont été considérés comme le problème majeur de la production des Cucurbitacées à travers le monde (Sevik et Arli-Sokmen, 2003 ; Lecoq et Desbiez, 2008; Ali et al., 2012a). Ces quatre virus sont transmis par des pucerons selon le mode non persistant et également transmissibles mécaniquement par la sève à des plantes (Mnari et al., 2008; Lecoq et Desbiez, 2008). Contrairement aux autres virus, la gamme d'hôtes du CMV est extrêmement large et distribuée mondialement avec plus de 1200 espèces végétales, représentant 100 familles botaniques incluant les Cucurbitacées et les Solanacées (Palukaitis et Garcia-Arenal, 2003 ; Jacquemond, 2012). La transmission du CMV par les semences a été rapportée avec des taux de transmission variables $(0-100 \%)$ chez certaines plantes incluant les plantes adventices (O'Keefe et al., 2007 ; Ali et Kobayaski, 2010; Salem et al., 2010). Papaya ringspot virus, WMV et ZYMV ont une gamme d'hôtes très étroite (Brunt et al., 1997). Outre Carica papaya (Caricacées) et quelques espèces de Chenopodiacées, une quinzaine d'espèces dans la famille des Cucurbitacées ont été signalées comme hôtes naturels du PRSV. Quant au WMV il peut infecter plusieurs espèces de légumineuses, la carotte (Carota dauci) et des espèces de mauvaises herbes (Parry et Persley, 2005 ; Ali et al., 2012b).

De nombreuses études ont été entreprises dans certains pays, notamment en Turquie, en Tunisie et aux Etats-Unis afin d'identifier les virus responsables de ces mosaïques et d'envisager des méthodes de lutte (Sevik et Arli-Sokmen, 2003; Mnari et al., 2008 ; Ali et al., 2012a). En Côte, les travaux de Fauquet et Thouvenel (1987) avaient permis d'identifier quelques virus produisant des mosaïques sur le concombre et la courgette. En 2010, Koné et collaborateurs ont confirmé la présence du CMV, du PRSV et signalé pour la première fois le ZYMV dans le sud de la Côte d'Ivoire. Cependant, aucune autre étude n'a été conduite pour décrire la situation phytosanitaire actuelle des concombres, courges et courgettes dans les principales zones de production. L'identification des virus responsables de ces symptômes de mosaïque dans des parcelles 
paysannes s'avère nécessaire afin d'envisager des stratégies de gestion intégrée et ainsi améliorer la productivité. C'est dans ce cadre que se situe cette étude dont l'objectif est d'actualiser les données relatives aux virus des concombres, courges et des courgettes dans différentes régions productrices.

\section{MATERIEL ET METHODES \\ Matériel végétal}

Le matériel végétal utilisé a été obtenu à partir des feuilles infectées naturellement de plantes de C. sativus, C. maxima et C. pepo cultivées dans des parcelles paysannes et montrant des symptômes typiques de viroses (Figure 1) en Côte d'Ivoire.

\section{Prospections, collecte et conservation des échantillons}

De juillet à octobre 2010, des prospections ont été réalisées en plein champ de Cucurbitacées dans les régions d'Abidjan, Bouaké, Daloa, Korhogo, Man, San Pedro et Yamoussoukro (Figure 2). Au total, 18 parcelles ont été visitées et 169 échantillons collectés. La collecte des échantillons s'est faite sur la base de la présence de symptômes de maladies virales. Parmi les 169 échantillons de Cucurbitacées collectés, 81 sont des concombres (C. sativus), 73 des courgettes (C. pepo) et 15 des courges $(C$. maxima). Lors de cette collecte, les symptômes observés ont été décrits et les feuilles présentant les symptômes photographiées. Deux ou trois feuilles ont été prélevées par plant malade selon le stade végétatif de la plante. Chaque isolat identifié a été conditionné par une déshydratation au chlorure de calcium selon la méthode de Bos (Mnari et al., 2008). Les rouleaux de feuilles découpées en lanières étaient déposés dans les pots de pellicules de photo contenant $3,5 \mathrm{~g}$ de chlorure de calcium. Les pots étaient conservés à la température ambiante $\left(25^{\circ} \mathrm{C}\right)$ jusqu'au retour de la collecte. Ils ont été acheminés au laboratoire de Phytopathologie et de Biologie Végétale de l'Institut National Polytechnique Félix Houphouët Boigny (INP$\mathrm{HB}$ ) de Yamoussoukro et maintenus à $4{ }^{\circ} \mathrm{C}$ au réfrigérateur avant la réalisation des tests sérologiques.

\section{Détection des virus recherchés par Double Antibody Sandwich-Enzyme Linked Immunosorbent Assay (DAS-ELISA) \\ Les échantillons de feuilles de} Cucurbitacées collectés ont été testés au Double Antibody Sandwich-Enzyme Linked Immunosorbent Assay (DAS-ELISA) selon le protocole de Clark et Adams (1974) comme rapporté également par Mnari et collaborateurs (2008) et en tenant compte des spécifications du fabriquant des kits (LOEWE Biochemica GmbH, Sauerlach, Allemagne) utilisés pour la confirmation de la présence des virus recherchés CMV, ZYMV, PRSV, WMV. Ces kits commerciaux ELISA comprenaient les anticorps spécifiques polyclonaux, des conjugués et des témoins positifs et négatifs ainsi que des tampons d'extraction, de sensibilisation et de lavage. Les échantillons de feuilles ont été broyés avec un extracteur manuel à billes (AGDIA, France). Le broyage a été réalisé avec un ratio de $0,2 \mathrm{~g}$ d'échantillon séché au $\mathrm{CaCl}_{2}$ pour 4 $\mathrm{ml}$ du tampon d'extraction. Le jus clarifié par décantation (maintenu sur de la glace) a alors été recueilli dans des pots pour une utilisation à l'étape de l'ajout de l'antigène.

Deux lectures des densités optiques ont été réalisées à $405 \mathrm{~nm}$ avec un spectrophotomètre ou lecteur de plaque (Multiskan plus version 2.01) une heure et deux heures après le dépôt du substrat de l'enzyme.

\section{Analyse des données sérologiques \\ Le seuil de positivité (SP) a été calculé avec des densités optiques (DO) à $405 \mathrm{~nm}$ des puits du contrôle négatif selon la formule de Sevik et Arli-Sokmen (2003): SP $=2 \times$ la moyenne des DO à $405 \mathrm{~nm}$ des puits ayant reçu le contrôle négatif. Les échantillons pour lesquels la moyenne des DO était supérieure au SP étaient déclarés positifs. Les échantillons négatifs au test DAS-ELISA étaient ceux pour lesquels la moyenne des DO était inférieure au SP.}




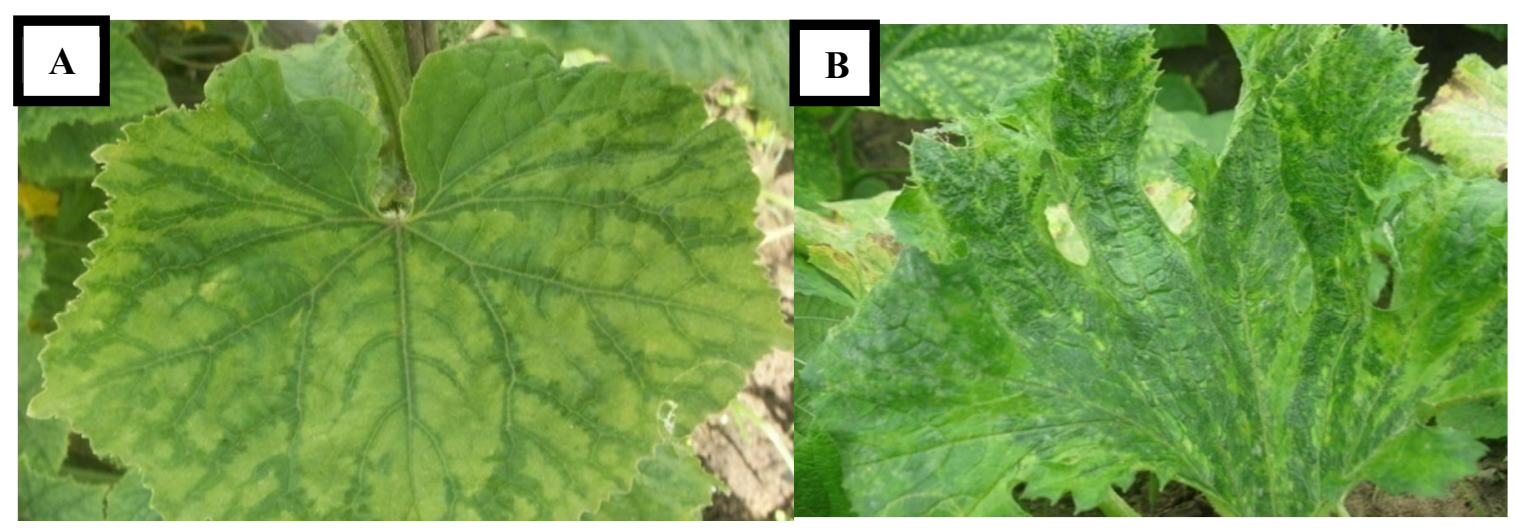

Figure 1 : Symptômes foliaires sur Cucurbitacées. A. Chlorose internervaire et liséré de nervure sur C. sativus ; B. Boursouflures, mosaïques et déformation foliaire sur C. maxima.

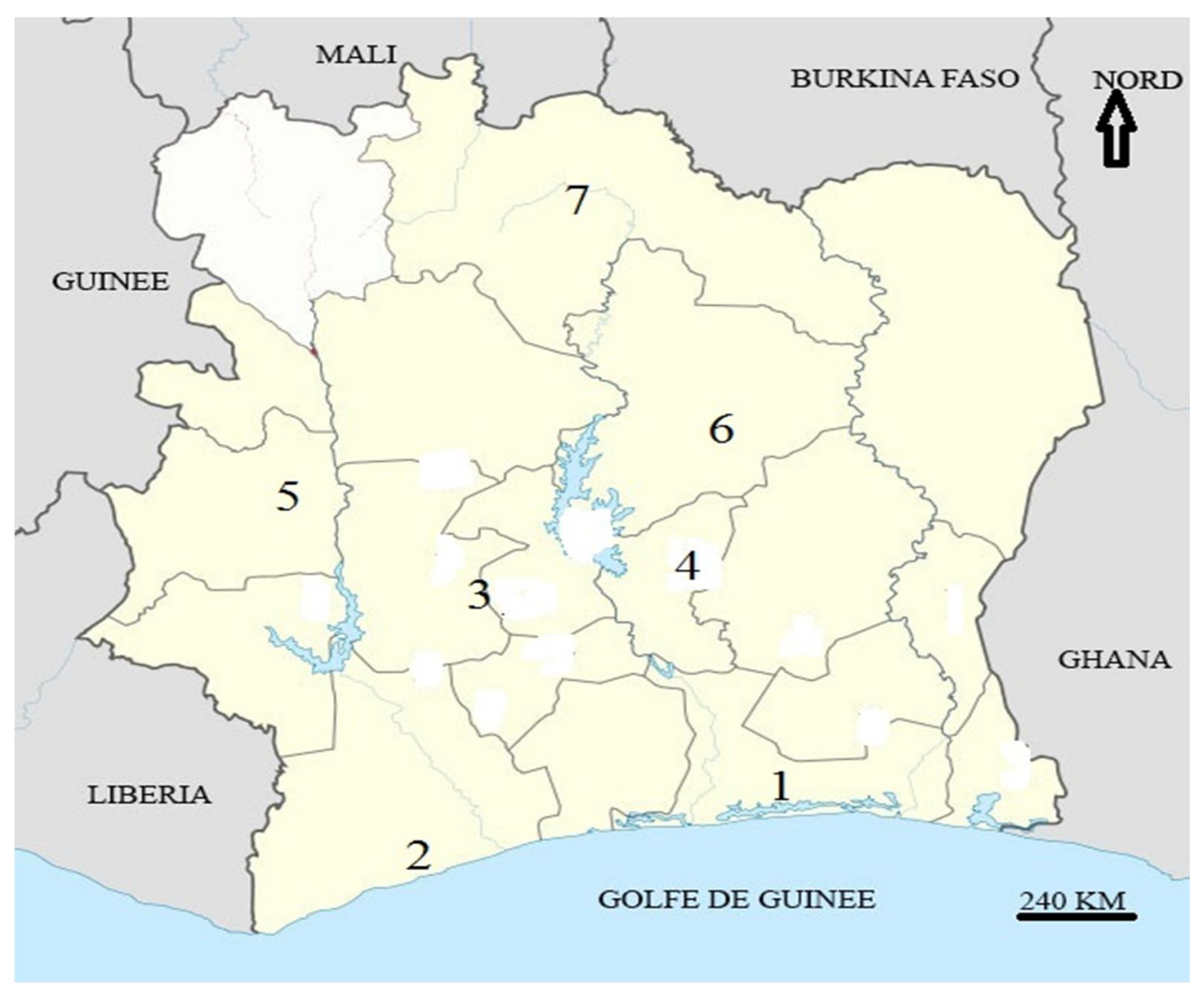

Figure 2 : Zones de collecte des échantillons de Cucurbitacées présentant des symptômes viraux en Côte d'Ivoire en 2010. Abidjan (1), San Pedro (2), Daloa (3), Yamoussoukro (4), Man (5), Bouaké (6) et Korhogo (7). 


\section{RESULTATS}

Les tests ont été réalisés en utilisant un contrôle négatif et un contrôle positif pour chaque virus provenant des kits commerciaux. Ainsi, la gamme des DO des contrôles négatifs a varié de 0,089 à 0,216 selon les antisera utilisés. De même, les échantillons positifs ont donné des valeurs d'absorbance de 0,211 à 2,775. Les résultats des tests sérologiques résumés au Tableau 1 montrent que les Cucurbitacées en Côte d'Ivoire sont infectées par le CMV, le PRSV, le WMV et le ZYMV. Sur les 169 échantillons de feuilles présentant des symptômes de viroses, les tests ont permis de confirmer l'infection de 59 échantillons par le CMV (soit un taux de détection de 34,91\%), de 18 échantillons par le PRSV (soit un taux de 10,65\%), de 18 échantillons par le WMV (soit un taux de $10,65 \%$ ) et finalement de 101 échantillons par le ZYMV (soit un taux de 59,76\%). L'ensemble des infections simples représentaient $47,93 \%$ (soit 81 sur 169) des échantillons testés avec 53 infectés par ZYMV (31,36\%), 23 par CMV (13,61\%), 3 par WMV $(1,78 \%)$ et 2 par PRSV $(1,18 \%)$. Les infections virales doubles étaient de $29,59 \%$ dont les combinaisons les plus importantes étaient celle de CMV+ZYMV, suivie par $\mathrm{WMV}+\mathrm{ZYMV}$, et finalement le CMV+PRSV avec respectivement 14,79\%; $7,10 \%$ et $3,55 \%$ des échantillons testés. Les infections triples $(5 / 169)$ présentes dans l'étude étaient celles de $\mathrm{CMV}+\mathrm{PRSV}+\mathrm{ZYMV}, \mathrm{CMV}+\mathrm{WMV}+\mathrm{ZYMV}$ et PRSV+WMV+ZYMV. En tout, le pourcentage de plantes infectées par au moins un des quatre virus (CMV, PRSV, WMV et ZYMV) est relativement très important d'une localité à une autre avec un minima de 61,3\% à San Pedro et un maxima de $100 \%$ à Yamoussoukro (Figure 3). Le ZYMV a été le virus le plus prédominant sur les cultures de C. sativus, de C. maxima et de C. pepo. Ce dernier a été détecté respectivement dans $55,5 \%(45 / 81), \quad 93,3 \%(14 / 15)$ et $57,5 \%$ (42/73) des échantillons testés (Figure 4). Parmi les échantillons de C. maxima testés, le ZYMV, le WMV et le PRSV se sont révélés présents avec respectivement $93,3 \% ; 26,6 \%$ et $6,6 \%$ des échantillons positifs. Le CMV a été le deuxième virus le plus important dans les échantillons de C. sativus et $C$. pepo avec respectivement $38,12 \%$ et $39,7 \%$ des échantillons positifs. Cucurbita maxima a été la culture la plus infectée avec un taux d'infection de $93,3 \%$, ensuite $C$. pepo et $C$. sativus avec respectivement $82,19 \%$ et $76,54 \%$ (Figure 4). Les parcelles en association culturale (avec d'autres Cucurbitacées ou non) présentent un taux d'infection de $83,70 \%$ contre $76,6 \%$ des échantillons infectés provenant des parcelles en monoculture (Tableau 2). Le ZYMV est très prédominant dans les associations culturales car il est présent dans tous les types d'infections (simples, doubles et triples) sur 66 parmi les 92 analysés, soit un taux d'infection de 71,74\%. En seconde position le CMV en infection simple, double $(\mathrm{CMV}+\mathrm{ZYMV})$ et triple $(\mathrm{CMV}+\mathrm{PRSV}+\mathrm{ZYMV})$, et présent sur 30/92 échantillons soit un taux de $32,61 \%$. Par contre en monoculture, les virus majeurs sont le CMV et le ZYMV avec respectivement $49,35 \%$ et $46,75 \%$ des échantillons analysés. 
K. T. KOUADIO et al. / Int. J. Biol. Chem. Sci. 11(3): 1113-1123, 2017

Tableau 1 : Bilan de la présence des virus dans les échantillons de Cucurbitacées collectés dans 7 régions de la Côte d'Ivoire en 2010.

\begin{tabular}{|c|c|c|c|c|c|c|c|c|c|}
\hline \multirow[t]{2}{*}{ Types d'infections } & \multicolumn{7}{|c|}{ Localités } & \multirow[t]{2}{*}{ Total } & \multirow[t]{2}{*}{$\%$ d'infections } \\
\hline & Abidjan & Bouaké & Daloa & Man & Korhogo & San Pedro & Yamoussoukro & & \\
\hline Nombre d'échantillons testés & 28 & 24 & 20 & 20 & 26 & 31 & 20 & 169 & ..... \\
\hline CMV & 7 & 3 & 1 & 6 & 0 & 6 & 0 & 23 & $13,6 \%$ \\
\hline PRSV & 0 & 0 & 0 & 2 & 0 & 0 & 0 & 2 & $1,2 \%$ \\
\hline WMV & 0 & 0 & 0 & 0 & 2 & 1 & 0 & 3 & $1,8 \%$ \\
\hline ZYMV & 2 & 9 & 14 & 2 & 13 & 7 & 6 & 53 & $31,3 \%$ \\
\hline Total infections simples & 9 & 12 & 15 & 10 & 15 & 14 & 6 & 81 & $47,9 \%$ \\
\hline $\mathrm{CMV}+\mathrm{PRSV}$ & 3 & 0 & 0 & 3 & 0 & 0 & 0 & 6 & $3,5 \%$ \\
\hline $\mathrm{CMV}+\mathrm{WMV}$ & 1 & 0 & 0 & 0 & 0 & 0 & 0 & 1 & $0,6 \%$ \\
\hline $\mathrm{CMV}+\mathrm{ZYMV}$ & 5 & 4 & 3 & 1 & 1 & 3 & 8 & 25 & $14,8 \%$ \\
\hline PRSV + WMV & 0 & 0 & 0 & 0 & 0 & 0 & 0 & 0 & $0 \%$ \\
\hline PRSV + ZYMV & 0 & 2 & 0 & 1 & 0 & 0 & 3 & 6 & $3,5 \%$ \\
\hline WMV + ZYMV & 0 & 2 & 0 & 0 & 8 & 2 & 0 & 12 & $7,1 \%$ \\
\hline Total infections doubles & 9 & 8 & 3 & 5 & 9 & 5 & 11 & 50 & $29,6 \%$ \\
\hline $\mathrm{CMV}+\mathrm{PRSV}+\mathrm{WMV}$ & 0 & 0 & 0 & 0 & 0 & 0 & 0 & 0 & $0 \%$ \\
\hline $\mathrm{CMV}+\mathrm{PRSV}+\mathrm{ZYMV}$ & 0 & 0 & 1 & 0 & 0 & 0 & 2 & 3 & $1,8 \%$ \\
\hline $\mathrm{CMV}+\mathrm{WMV}+\mathrm{ZYMV}$ & 0 & 0 & 0 & 0 & 1 & 0 & 0 & 1 & $0,6 \%$ \\
\hline $\mathrm{PRSV}+\mathrm{ZYMV}+\mathrm{WMV}$ & 0 & 0 & 0 & 0 & 0 & 0 & 1 & 1 & $0,6 \%$ \\
\hline Total infection triples & $\mathbf{0}$ & $\mathbf{0}$ & 1 & $\mathbf{0}$ & 1 & $\mathbf{0}$ & 3 & 5 & $3,0 \%$ \\
\hline $\mathrm{CMV}+\mathrm{PRSV}+\mathrm{WMV}+\mathrm{ZYMV}$ & 0 & 0 & 0 & 0 & 0 & 0 & 0 & 0 & $0.00 \%$ \\
\hline Total échantillons positifs & 18 & 20 & 19 & 15 & 25 & 19 & 20 & 136 & $80,5 \%$ \\
\hline Total échantillons négatifs aux 4 virus & 10 & 4 & 1 & 5 & 1 & 12 & $\mathbf{0}$ & 33 & $19,5 \%$ \\
\hline
\end{tabular}




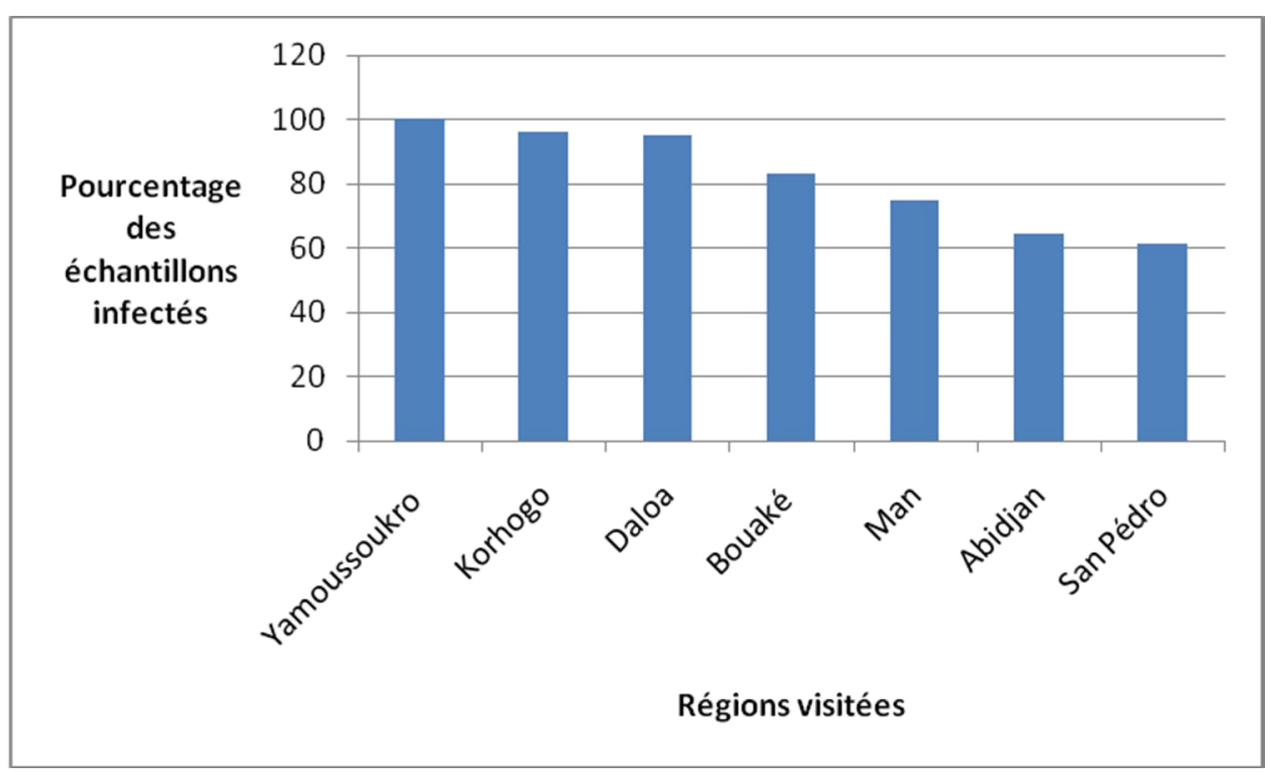

Figure 3 : Pourcentage des échantillons de Cucurbitacées infectés par au moins un virus dans 7 régions visitées en Côte d'Ivoire en 2010.

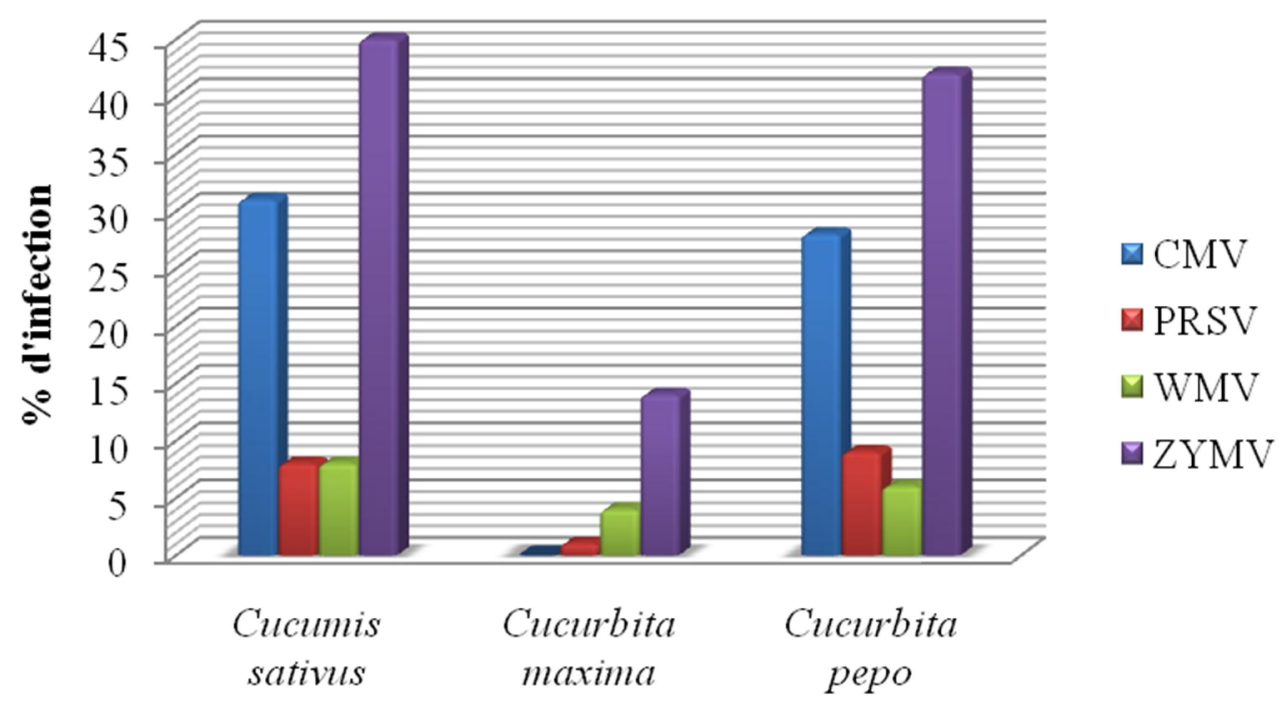

Figure 4 : Présence du CMV, PRSV, WMV et ZYMV sur les espèces de Cucurbitacées échantillonnées en Côte d'Ivoire en 2010. 
Tableau 2 : Présence des virus dans les parcelles visitées en Côte d'Ivoire en 2010 selon le mode de culture.

\begin{tabular}{lccc}
\hline Types d'infection présents & \multicolumn{2}{c}{ Mode de culture } & Total \\
\cline { 2 - 3 } & Monoculture & En association & \\
\hline Nombre d'échantillons testés & $\mathbf{7 7}$ & $\mathbf{9 2}$ & $\mathbf{1 6 9}$ \\
CMV & 13 & 9 & $\mathbf{2 2}$ \\
PRSV & 2 & 0 & $\mathbf{2}$ \\
WMV & 1 & 2 & $\mathbf{3}$ \\
ZYMV & 16 & 38 & $\mathbf{5 4}$ \\
CMV+PRSV & 6 & 0 & $\mathbf{6}$ \\
CMV+WMV & 1 & 0 & $\mathbf{1}$ \\
CMV+ZYMV & 7 & 18 & $\mathbf{2 5}$ \\
PRSV+ZYMV & 2 & 4 & $\mathbf{6}$ \\
WMV+ZYMV & 10 & 2 & $\mathbf{1 2}$ \\
CMV+PRSV+ZYMV & 0 & 3 & $\mathbf{3}$ \\
CMV+WMV+ZYMV & 1 & 0 & 1 \\
PRSV+WMV+ZYMV & 0 & 1 & $\mathbf{1}$ \\
Total & $\mathbf{5 9}$ & $\mathbf{7 7}$ & $\mathbf{1 3 6}$ \\
\% d'infections & $\mathbf{7 6 , 6 \%}$ & $\mathbf{8 3 , 7 0 \%}$ \\
\hline
\end{tabular}

Cucumber mosaic virus (CMV), Papaya ringspot virus (PRSV), Watermelon mosaic virus (WMV) et Zucchini yellow mosaic virus (ZYMV).

\section{DISCUSSION}

Les résultats de cette étude menée sur des échantillons collectés lors de l'enquête réalisée en 2010 montrent bien que le CMV, le PRSV, le WMV et le ZYMV infectent les Cucurbitacées dans des conditions de pleins champs en Côte d'Ivoire. Ils confirment la présence du CMV et du PRSV déjà rapportés par Fauquet et Thouvenel en 1987. Ces auteurs ont réussi à isoler le CMV dans le secteur mésophylle et l'ont transmis de façon mécanique à une large gamme d'hôtes et par les pucerons sous le mode non persistant. Quant au PRSV, il a été identifié dans les régions Centre et Sud de la Côte d'Ivoire et sa transmission s'est faite mécaniquement et par les pucerons (Aphis gossypii) sous le mode non-persistant. Ces résultats confirment également ceux de Koné et al. (2010) qui ont montré que ZYMV était présent dans des échantillons collectés (4 des 6 échantillons de courgettes) au cours d'une enquête de décembre 2008 à janvier 2009 dans les villes d'Adzopé, Songon, Ayamé et Gagnoa. Aussi, ils ont observé, en plus de la présence du CMV, celle du PRSV et des infections virales doubles $(\mathrm{CMV}+\mathrm{PRSV})$ dans les échantillons de concombre. De même, Diallo et al. (2007) ont signalé la présence du PRSV en Côte d'Ivoire sur le papayer lors d'une étude menée dans le district d'Abidjan. L'étude d'Agneroh et al. (2012) a également rapporté l'infection de Lagenaria siceraria et Citrullus sp. par le CMV, PRSV et ZYMV dans trois localités de la Côte d'Ivoire (Korhogo, Bouaké et Yamoussoukro). Ainsi, cette présente étude rapporte pour la première fois la présence du WMV parmi les virus infectant les 
Cucurbitacées en Côte d'Ivoire. Ce virus a été trouvé dans les échantillons provenant des régions d'Abidjan, de Bouaké, de Korhogo, de San Pedro et de Yamoussoukro. Il serait alors opportun de compléter cette étude par une caractérisation moléculaire des isolats de WMV présents en Côte d'Ivoire. Kenwyn et al. (2002) ont identifié le WMV avec comme taux d'infection de $22,6 \%$ dans des échantillons de Cucurbitacées collectés dans la région de Kwazulu-Natal (Afrique du Sud). De même, Yuri et al. (2000) ont signalé la présence de WMV avec un taux d'infection de l'ordre de $10,65 \%$ dans une étude menée à Sao Paulo (Brésil). Dans cette étude menée en Côte d'Ivoire, le WMV apparaît comme l'un des virus (avec le PRSV) le moins répandu dans les plantes infectées $(10,6 \%)$; ce qui pourrait s'expliquer par le fait que ce virus n'est pas assez fréquent dans les zones tropicales (Shoeibi et al., 2009). Cependant, il serait souhaitable que d'autres prospections soient faites en Côte d'Ivoire parce que ce virus est présent dans 5 des 7 localités visitées. Le ZYMV a été le virus le plus répandu parmi les échantillons étudiés et cette fréquence élevée pourrait gravement réduire la production des Cucurbitacées à travers le pays car il est présent dans les 7 localités visitées. De même, les prospections menées de 1993 à 1995 par Mahgoub et al. (1997) ont révélé que le ZYMV est le virus le plus prédominant sur les Cucurbitacées au Soudan.

Des infections mixtes à diverses fréquences impliquant $\mathrm{CMV}+\mathrm{ZYMV}$, WMV+ZYMV, CMV+PRSV, PRSV+ ZYMV, et CMV+WMV ont été rencontrées dans toutes les zones d'études. Egalement des études semblables ont révélé des infections mixtes telles l'infection double WMV+ZYMV de $16,7 \%$ sur le melon en Turquie (Gassan et Yilmaz, 2006). En observant les résultats de cette étude, il semble que ce mode d'infection mixte soit aléatoire et ne soit pas lié à une zone géographique spécifique. Nous ne pouvons pour l'instant rien affirmer mais des études approfondies en saison sèche comme en saison pluvieuse pourraient nous dire avec précision si ceci est lié au plan agroécologique de la zone, au mode de transmission de ces virus ou à leurs propriétés. Trente-trois (33) échantillons présentant des symptômes viraux ont été testés négatifs aux quatre antisera. Ceci pourrait être dû à d'autres virus non pris en compte dans le cadre de cette étude. Ces échantillons présentaient des symptômes typiques de maladies virales comme des mosaïques, des boursouflures, des lisérés des nervures et des plages chlorotiques. On pourrait les attribuer à certains virus déjà testés par Kone et al. (2010) mais non confirmés ou d'autres identifiés en Tunisie (Mnari-Hattab et al., 2008), au Brésil par Yuki et al. (2000). Dans d'autres cas, le titre viral pourrait être en-deçà du seuil détectable par la sérologie, donc il serait aussi opportun de faire des tests moléculaires.

\section{Conclusion}

L'étude menée dans le cadre de ce travail avait pour objectif d'actualiser les données sur les virus responsables des mosaïques chez les Cucurbitacées en Côte d'Ivoire. L'identification de ces virus permet de mieux connaître leur épidémiologie et de mettre au point des stratégies de lutte plus efficaces afin de limiter leur impact sur la production. Ces tests ont permis de confirmer la présence de trois virus parmi les quatre qui ont déjà été signalés et d'identifier pour la première fois le WMV. Néanmoins, il ne faut pas perdre de vue que certains échantillons ont été testés négatifs malgré la présence de symptômes viraux, ce qui amène à continuer l'étude en utilisant d'autres antiséra et/ou des outils de détection complémentaires comme les tests moléculaires.

\section{CONFLIT D'INTERETS}

Les auteurs de ce travail déclarent qu'il n'existe aucun conflit d'intérêt. 


\section{CONTRIBUTIONS DES AUTEURS}

Tous les auteurs ont contribué à la collecte des échantillons, à leur analyse sérologique et à la rédaction de ce manuscrit.

\section{REMERCIEMENTS}

Les auteurs remercient tous les paysans des localités visitées ayant accepté des prospections dans leurs parcelles incluant des prélèvements d'échantillons. En outre, nous remercions Dr Mnari-Hattab Monia du Laboratoire de Biotechnologie Appliquée à l'Agriculture, INRA Tunisie, pour sa contribution à l'amélioration de ce manuscrit.

\section{REFERENCES}

Ali A, Kobayashi M. 2010. Seed transmission of Cucumber mosaic virus in pepper. $J$. Virol. Methods, 163: 234-237. Doi: 10.1016/j.jviromet.2009.09.026.

Ali A, Abdalla O, Bruton B, Fish W, Sikora E, Zhang S, Taylor M. 2012b. Occurrence of viruses infecting watermelon, other cucurbits, and weeds in the parts of southern United States. Online. P. Health Progr., Doi: 10.1094/PHP-2012-0824-01-RS.

Ali A, Mohammad O, Khattab A. 2012a. Distribution of viruses infecting cucurbit crops and isolation of potential new virus-like sequences from weeds in Oklahoma. Plant Dis., 96: 243-248. Doi: 10.1094 / PDIS-05-11-0419.

Ali A, Natsuaki T, Okuda S. 2004. Identification and molecular characterization of viruses infecting cucurbits in Pakistan. J. Phytopathology, 152: 677-682. DOI: $10.1111 / \mathrm{j} .1439-$ 0434.2004.00915.x

ANADER. 2004. Rapport d'activité 2003, Zone d'Abengourou. 82p.

Brunt AA, Crabtree K, Dallwitz MJ, Gibbs AJ, Watson L, Zurcher EJ. 1997. Plant Viruses Online: Descriptions and lists from the VIDE database. Version $16^{\text {th }}$ January.
Diallo AH, Monger W, Kouassi KN, Yoro DT, Jones P. 2007. First report of Papaya ringspot virus (PRSV) infecting papaya in Côte d'Ivoire. Plant Pathol., 56(4): 718 . DOI: $10.1111 / \mathrm{j} .1365-$ 3059.2007.01593.x

FAO. 2009. Rapport national sur l'état des ressources phytogénétiques pour l'alimentation et l'agriculture. Second rapport national. Ministère de l'Agriculture (République de Côte d'ivoire), $65 \mathrm{p}$.

Fauquet C, Thouvenel JC. 1987. Maladies Virales des Plantes en Côte d'Ivoire/Plant viral Diseases in the Ivory Coast. Ed ORSTOM : Paris ; 183-184.

Gassan K, Yilmaz O. 2006. Occurrence of Cucurbit viruses on field-grown melon and watermelon in the Thrace region of Turkey. Phytoprotection, 87(3): 123130.

Jacquemond M. 2012. Cucumber mosaic virus. Adv. Virus Res., 84: 439-504.

Kenwyn RC, John VDG, Mark DL. 2001. Virus infecting cucurbits in KwazuluNatal, South Africa. Rev. Mex. Fitopathol., 19: 251-252.

Koné D, Aké S, Abo K, Soro S, N'Guessan CA, Wipf-Scheibel C, Chandeysson C, Desbiez C, Lecoq H. 2010. First Report of Zucchini yellow mosaic virus in Cucurbits in Ivory Coast. Plant Dis., 94(11): 1378. DOI: 10.1094/PDIS-0610-0416.

Lecoq H, Desbiez C. 2008. Watermelon mosaic virus and Zucchini yellow mosaic virus. In Encyclopedia of Virology (vol. 5, $3^{\text {rd }}$ edn), Mahy BWJ, Van Regenmortel MHV (eds). Elsevier: Oxford; 433-440.

Mahgoub AH, Desbiez C, Wipt-Scheibel C, Dafalla G, Lecoq H. 1997. Characterization and occurrence of Zucchini Yellow Mosaic Virus in Sudan. Plant Pathol., 46: 800-805.

Mnari HM, Jebari H, Zouba A. 2008. Identification et distribution des virus 
responsables de mosaïques chez les Cucurbitacées en Tunisie. Bulletin OEPP/EPPO, Bulletin, 38: 497-500.

O'Keefe DC, Berryman DI, Coutts BA, Jones RAC. 2007. Lack of seed coat contamination with cucumber mosaic virus in lupin permits reliable, largescale detection of seed transmission in seed samples. Plant Dis., 91: 504-508. Doi: 10.1094/PDIS-91-5-0504.

Palukaitis P, Garcia-Arenal F. 2003. Cucumoviruses. Adv. Virus Res., 62: 241-323.

Parry JN, Persley DM. 2005. Carrot as a natural host of Watermelon mosaic virus. Austr. Plant Pathol., 34: 283-284. DOI: 10.1071/AP05016.

Provvidenti R. 2010. Diseases caused by viruses. In Compendium of Cucurbit Diseases, Zitter TA, Hopkins DL, Thomas CE (eds). American Phytopathological Society, St. Paul: Minnesota; 37-45.

Salem NM, Ehlers JD, Roberts PA, Ng JCK. 2010. Biological and molecular diagnosis of seedborne viruses in cowpea germplasm of geographically diverse sub-saharan origins. Plant Pathol., 59:
773-784. DOI: $10.1111 / \mathrm{j} .1365-$ 3059.2010.02285.x

Sevik MA, Arli S. 2003. Virus infecting Cucurbits in Samsun, Turkey. Plant Dis., 87: 341-344. DOI: 10.1094/PDIS.2003.87.4.341

Shoeibi S, Masumi M, Nasrollanezhad S, Heydari S, Izadpanah K, Ahmadikhah A. 2009. Sequencing of six Iranian isolates of watermelon mosaic virus and phylogenetic comparison of Iranian isolates with other isolates of the world. Iran. J. Plant Pathol., 45(2): 34-37.

Verma R, Baranwal VK, Prakash S, Tomer SPS, Singh J, Pant RP, Ahlawat YS. 2006. Biological and molecular characterization of Zucchini yellow mosaic virus from naturally infected bottle gourd. Ind. J. Virol., 17(2): 96101.

Yuki VA, Rezende JAM, Kitajima EW, Barroso PAV, Kuniyuki H, Groppo GA, Pavan MA. 2000. Occurrence, distribution and relative incidence of five viruses infecting cucurbits in the state of São Paulo, Brazil. Plant Dis., 84: 516520. DOI: 10.1094/PDIS.2000.84.5.516 